\title{
Ergodic theorems and perturbations of contraction semigroups
}

\author{
by \\ MARTA Tyran-Kamińska (Katowice)
}

\begin{abstract}
We provide sufficient conditions for sums of two unbounded operators on a Banach space to be (pre-)generators of contraction semigroups. Necessary conditions and applications to positive emigroups on Banach lattices are also presented.
\end{abstract}

1. Introduction. Let $X$ be a Banach space, $A: \mathcal{D}(A) \subseteq X \rightarrow X$ be a densely defined linear operator with a nonempty resolvent set $\rho(A)$, and let $B: \mathcal{D}(B) \subseteq X \rightarrow X$ be a linear operator such that $\mathcal{D}(B) \supseteq \mathcal{D}(A)$. If the operator $(A+B, \mathcal{D}(A))$ is dissipative then by the Lumer-Phillips theorem [10] the closure of the operator $A+B$ is the (infinitesimal) generator of a $C_{0}$-semigroup of contractions if and only if the range of the operator $\lambda-A-B$ is dense in $X$ for some $\lambda>0$. The verification of the range condition is not an easy task and makes the theorem hard to apply.

In this paper we provide generation results in terms of ergodic properties of the operator $B R(\lambda, A)$, where we let $R(\lambda, A):=(\lambda-A)^{-1}$ be the resolvent of $A$. A linear bounded operator $T$ on $X$ is called mean ergodic if the Cesàro means

$$
\frac{1}{n} \sum_{k=0}^{n-1} T^{k} \text { converge strongly in } X,
$$

and uniformly ergodic if the means converge in the uniform operator topology. Our main result is the following

TheOrem 1.1. Let $(A, \mathcal{D}(A))$ be a densely defined linear operator in a Banach space $X$ such that $\rho(A)_{+}:=\rho(A) \cap(0, \infty) \neq \emptyset$. Suppose that $(A+B, \mathcal{D}(A))$ is dissipative and $B R(\lambda, A)$ is mean ergodic for some $\lambda \in$ $\rho(A)_{+}$. Then the closure of $(A+B, \mathcal{D}(A))$ generates a $C_{0}$-semigroup of con-

2010 Mathematics Subject Classification: 47A35, 47B44, 47B60, 47D06.

Key words and phrases: $C_{0}$-semigroup of contractions, perturbation of semigroups, positive semigroups, generators, dissipative operators, mean ergodic, power bounded, quasicompact. 
tractions on $X$. Moreover, if $B R(\lambda, A)$ is uniformly ergodic then $(A+B, \mathcal{D}(A))$ is closed.

Recall that a linear operator $T$ is called power bounded if $\sup _{n}\left\|T^{n}\right\|<\infty$, and quasi-compact if there exists a compact operator $K$ and $m \in \mathbb{N}$ such that $\left\|T^{m}-K\right\|<1$. By the Yosida-Kakutani uniform ergodic theorem [14], every power bounded and quasi-compact operator is uniformly ergodic. Since power bounded operators on reflexive Banach spaces are mean ergodic, we obtain the following perturbation result from Theorem 1.1.

Corollary 1.2. Let $(A+B, \mathcal{D}(A))$ be densely defined and dissipative. Assume that for some $\lambda \in \rho(A)_{+}$the operator $B R(\lambda, A)$ is power bounded.

(i) If $B R(\lambda, A)$ is quasi-compact, then $(A+B, \mathcal{D}(A))$ generates a $C_{0}$ semigroup of contractions.

(ii) If $X$ is reflexive then the closure of $(A+B, \mathcal{D}(A))$ generates a $C_{0}$ semigroup of contractions.

Theorem 1.1 and its partial converse (Theorem 3.3) are proved in Section 3. Let us now indicate how some perturbation results are related to our result. The operator $B$ is (relatively) A-bounded if there exist nonnegative constants $a, b$ such that

$$
\|B x\| \leq a\|A x\|+b\|x\|
$$

for all $x \in \mathcal{D}(A)$; the infimum of all possible $a$ is called the relative $A$-bound of $B$. If the $A$-bound is sufficiently small so that $\|B R(\lambda, A)\|<1$ for some $\lambda$ then $B R(\lambda, A)$ is power bounded and quasi-compact. This is the case for example when $A$ is a generator and $B$ is a bounded operator, or when $A$ is the generator of a semigroup of contractions and $B$ is $A$-bounded with $A$-bound less than $1 / 2$ (note that we do not require that $B$ is dissipative). For examples of operators with a small $A$-bound see Chapter III of [5].

Now we let $X$ be an ordered Banach space with a normal and generating cone $X_{+}$, i.e., $X=X_{+}-X_{+}, X^{*}=X_{+}^{*}-X_{+}^{*}$, where $X^{*}$ denotes the topological dual with the duality pairing $\langle\cdot, \cdot\rangle$. The order in $X$ and $X^{*}$ will be denoted by $\geq$. A linear operator $A$ is said to be positive if $A x \geq 0$ for $x \in \mathcal{D}(A)_{+}:=\mathcal{D}(A) \cap X_{+}$. A semigroup $\{S(t)\}_{t \geq 0}$ is called positive if every operator $S(t)$ is positive. In Section 4 we show that if $A$ and $A+B$ generate positive $C_{0}$-semigroups and the operator $(B, \mathcal{D}(A))$ is positive then $B R(\lambda, A)$ is power bounded and quasi-compact for all sufficiently large $\lambda$, which provides a converse to Corollary 1.2(ii).

Yet another consequence of our Theorem 1.1 is a generalization of $[4$, Theorem 3.2], proved in Section 4.

Theorem 1.3. Let $X$ be a real Banach lattice. Assume that the operators $(A, \mathcal{D}(A))$ and $(B, \mathcal{D}(B))$ in $X$ satisfy: 
(G1) $(A, \mathcal{D}(A))$ generates a $C_{0}$-semigroup of positive contractions on $X$;

$(\mathrm{G} 2) \mathcal{D}(B) \supseteq \mathcal{D}(A)$ and $B x \geq 0$ for $x \in \mathcal{D}(A)_{+}$;

(G3) for every $x \in \mathcal{D}(A)_{+}$there is $x^{*} \in X_{+}^{*}$ with $\left\|x^{*}\right\| \leq 1$ such that $\left\langle x^{*}, x\right\rangle=\|x\|$ and

$$
\left\langle x^{*}, A x+B x\right\rangle \leq 0 .
$$

If for some $\lambda>0$ the operator $B R(\lambda, A)$ is mean ergodic then the closure of $(A+B, \mathcal{D}(A))$ generates a $C_{0}$-semigroup of positive contractions.

In [4] it is assumed that the Banach lattice is a KB-space (KantorovichBanach space), i.e., every increasing norm bounded sequence of elements of the positive cone $X_{+}$is norm convergent, and that (G1)-(G3) hold together with $r(B R(\lambda, A)) \leq 1$ for some $\lambda>0$, where $r$ denotes the spectral radius. The conclusion of [4, Theorem 3.2] is that there is a generator $C$ of a $C_{0^{-}}$ semigroup of positive contractions which is an extension of the operator $(A+B, \mathcal{D}(A))$. Our theorem removes the restriction on the lattice being a KB-space, but the assumption on $B R(\lambda, A)$ is stronger. In the case of a KBspace, we obtain not merely the existence of a generator extending $A+B$, but identify it (under our stronger condition) as the closure of $(A+B, \mathcal{D}(A))$.

Finally, let us suppose that the operator $B R(\lambda, A)$ is a contraction for some $\lambda>0$. This can be achieved for example if $X=L^{1}(E, \mathcal{E}, m$ ) (see e.g. [4, Corollary 3.13]), where $(E, \mathcal{E}, m)$ is a $\sigma$-finite measure space, or [4, Remark 3.3] if $-A$ is a positive operator and

$$
\|B x\| \leq\|A x\|, \quad x \in \mathcal{D}(A)_{+} .
$$

Then from Theorems 1.3 and 3.3 it follows that when (G1)-(G3) hold, then the closure of $(A+B, \mathcal{D}(A))$ is a generator if and only if $B R(\lambda, A)$ is mean ergodic. As a corollary we obtain the following characterization.

Corollary 1.4. Let $X=L^{1}(E, \mathcal{E}, m)$ and the operators $(A, \mathcal{D}(A))$ and $(B, \mathcal{D}(B))$ satisfy $(\mathrm{G} 1)-(\mathrm{G} 2)$. Suppose that for all $x \in \mathcal{D}(A)_{+}$,

$$
\int_{E}(A x+B x) d m \leq 0 .
$$

(i) The operator $(A+B, \mathcal{D}(A))$ generates a $C_{0}$-semigroup of positive contractions if and only if $B R(\lambda, A)$ is quasi-compact for some $\lambda>0$.

(ii) The closure of $(A+B, \mathcal{D}(A))$ generates a $C_{0}$-semigroup of positive contractions if and only if $B R(\lambda, A)$ is strongly stable for some $\lambda>0$, i.e.,

$$
\lim _{n \rightarrow \infty}\left\|(B R(\lambda, A))^{n} x\right\|=0 \quad \text { for all } x \in L^{1}(E, \mathcal{E}, m) .
$$

Part (i) seems to be new and contains the particular cases from [6]. The equivalence in (ii) first appeared in [7] in the case of an $l_{1}$ space and was generalized by numerous authors (see [3] for a detailed exposition). 
By (G1), $R(\lambda, A)$ is positive for $\lambda>0$, so (G2) implies that $B R(\lambda, A)$ is a positive operator. Since the positive operator $B R(\lambda, A)$ has no nonzero fixed points, strong stability is equivalent to mean ergodicity by additivity of the $L^{1}$ norm. Other conditions equivalent to strong stability can be found in [12, Proposition 2.1].

2. Preliminaries. Let $X$ be a Banach space. We will denote by $\mathcal{D}(A)$ the domain of the operator $A$, by $\operatorname{Im}(A)$ its image, by $\operatorname{Ker}(A)$ its kernel (null space), and by $\sigma(A)$ its spectrum. The spectral bound of $A$ is defined as $s(A)=\sup \{\operatorname{Re} \lambda: \lambda \in \sigma(A)\}$ if the supremum exists $(s(A)=-\infty$ if $\sigma(A)$ is empty). If $A$ is a bounded operator then $r(A)$ denotes the spectral radius of $A$ and we have $r(A)=\lim _{n \rightarrow \infty} \sqrt[n]{\left\|A^{n}\right\|}$.

We say that $A \subseteq C$, or that $C$ is an extension of $A$, if $\mathcal{D}(A) \subseteq \mathcal{D}(C)$ and $C x=A x$ for $x \in \mathcal{D}(A)$. The operator $A$ is said to be closable if it has a closed extension. If $A$ is closable, then the closure $\bar{A}$ of $A$ is the minimal closed extension of $A$; more specifically, it is the closed operator whose graph is the closure in $X \times X$ of the graph of $A$. The operator $A$ is dissipative if $\|\lambda x-A x\| \geq \lambda\|x\|$ for all $x \in \mathcal{D}(A)$ and $\lambda>0$. If $A$ is a densely defined dissipative operator, then $A$ is closable and $\overline{\operatorname{Im}(\lambda I-A)}=\operatorname{Im}(\lambda I-\bar{A})$ for every $\lambda>0$. For the semigroup theory we refer to [5].

A bounded linear operator $T$ on $X$ is called Cesàro bounded if

$$
\sup _{N \geq 1} \frac{1}{N}\left\|\sum_{n=0}^{N-1} T^{n}\right\|<\infty .
$$

The following result is well known as the mean ergodic theorem $[8$, Theorem 2.1.3].

Theorem 2.1. Let $T$ be a Cesàro bounded operator on $X$ satisfying

$$
\lim _{n \rightarrow \infty} \frac{1}{n}\left\|T^{n} x\right\|=0 \quad \text { for every } x \in X .
$$

Then

$$
\left\{x \in X: \lim _{n \rightarrow \infty} \frac{1}{n} \sum_{k=0}^{n-1} T^{k} x \text { exists }\right\}=\operatorname{Ker}(I-T) \oplus \overline{\operatorname{Im}(I-T)}
$$

and for any $x \in X$ we have $x \in \overline{\operatorname{Im}(I-T)}$ if and only if

$$
\lim _{n \rightarrow \infty} \frac{1}{n} \sum_{k=0}^{n-1} T^{k} x=0 .
$$

If $T$ is mean ergodic, then it is Cesàro bounded by the Banach-Steinhaus theorem; it also satisfies (2) since 


$$
\frac{T^{n} x}{n}=\frac{n+1}{n} \frac{1}{n+1} \sum_{k=0}^{n} T^{k} x-\frac{1}{n} \sum_{k=0}^{n-1} T^{k} x \rightarrow 0 .
$$

Hence we obtain the following

Corollary 2.2. Let $T$ be a bounded linear operator on $X$. Then $T$ is mean ergodic and $\operatorname{Ker}(I-T)=\{0\}$ if and only if $T$ is Cesàro bounded, condition (2) holds, and $X=\overline{\operatorname{Im}(I-T)}$.

REMARK 2.3. If the operator $T$ is Cesàro bounded, then $r(T) \leq 1$, since

$$
\left\|T^{n}\right\| \leq(2 n+1) \sup _{N \geq 1} \frac{1}{N}\left\|\sum_{k=0}^{N-1} T^{k}\right\|, \quad n \geq 1 .
$$

From the uniform ergodic theorem [9, Theorem 1] and its proof we obtain the following result.

TheOREM 2.4. Let $T$ be a bounded linear operator on $X$. Then $T$ is uniformly ergodic if and only if $\left\|T^{n}\right\| / n \rightarrow 0$ as $n \rightarrow \infty$ and $\operatorname{Im}(I-T)$ is closed. Moreover, if $T$ is uniformly ergodic and $\operatorname{Ker}(I-T)=\{0\}$, then $1 \in \rho(T)$.

3. Perturbations in Banach spaces. In this section we assume that $(A, \mathcal{D}(A))$ and $(B, \mathcal{D}(B))$ are linear operators in a Banach space $X$ such that $\mathcal{D}(A) \subseteq \mathcal{D}(B)$ and

$$
\rho(A)_{+}:=\rho(A) \cap(0, \infty) \neq \emptyset .
$$

Lemma 3.1. Let $\lambda \in \rho(A)$ and let $B R(\lambda, A)$ be a bounded operator on $X$. Then the operator $(A+B, \mathcal{D}(A))$ satisfies

$$
\operatorname{Im}(\lambda I-A-B)=\operatorname{Im}(I-B R(\lambda, A)) .
$$

Moreover, $\lambda \in \rho(A+B)$ if and only if $1 \in \rho(B R(\lambda, A))$. In that case,

$$
R(\lambda, A+B)=R(\lambda, A)(I-B R(\lambda, A))^{-1} .
$$

Proof. Since $\lambda \in \rho(A)$, we have $\operatorname{Im}(R(\lambda, A))=\mathcal{D}(A)$ and $(\lambda-A) R(\lambda, A) x$ $=x$ for $x \in X$, which gives

$$
(\lambda I-A-B) R(\lambda, A) x=(I-B R(\lambda, A)) x \quad \text { for all } x \in X .
$$

Thus, the first assertion follows. We also have

$$
(\lambda I-A-B)=(I-B R(\lambda, A))(\lambda I-A),
$$

which together with (3) proves the claim.

Lemma 3.2. If $(A+B, \mathcal{D}(A))$ is closable, then $B R(\lambda, A)$ is a bounded operator on $X$ for all $\lambda \in \rho(A)$.

Proof. Let $\lambda \in \rho(A)$. We have $A+B \subseteq \overline{A+B}$, thus, for all $x \in X$,

$$
(A+B) R(\lambda, A) x=\overline{A+B} R(\lambda, A) x,
$$


which implies that

$$
B R(\lambda, A) x=\overline{A+B} R(\lambda, A) x+x-\lambda R(\lambda, A) x .
$$

Since $R(\lambda, A)$ is continuous and $\overline{A+B}$ is closed, it follows that the operator $B R(\lambda, A)$ has a closed graph, so it is bounded.

Proof of Theorem 1.1. Since the operator $(A+B, \mathcal{D}(A))$ is dissipative, it is closable, so $B R(\lambda, A)$ is a bounded operator for all $\lambda \in \rho(A)$. Moreover, (since $A+B$ is dissipative) for $\lambda \in \rho(A)_{+}$we have, by (3),

$$
\|x-B R(\lambda, A) x\|=\|(\lambda I-A-B) R(\lambda, A) x\| \geq \lambda\|R(\lambda, A) x\|
$$

for all $x \in X$. Hence, if $x \in \operatorname{Ker}(I-B R(\lambda, A))$, then $\|R(\lambda, A) x\|=0$, which shows that $x=0$ and $\operatorname{Ker}(I-B R(\lambda, A))=\{0\}$ for every $\lambda \in \rho(A)_{+}$. From Corollary 2.2 and Lemma 3.1 it follows that $\operatorname{Im}(\lambda I-A-B)$ is dense in $X$ for some $\lambda>0$. Thus, the claim follows from the Lumer-Phillips theorem.

If $B R(\lambda, A)$ is uniformly ergodic, then $\operatorname{Im}(\lambda I-A-B)$ is closed in $X$, by Theorem 2.4. Hence the operator $(A+B, \mathcal{D}(A))$, being dissipative, is closed.

We conclude this section with a partial converse of Theorem 1.1.

TheOREM 3.3. If the closure of $(A+B, \mathcal{D}(A))$ generates a $C_{0}$-semigroup of contractions and $B R(\lambda, A)$ is power bounded for some $\lambda \in \rho(A)_{+}$, then $B R(\lambda, A)$ is mean ergodic. Moreover, if $(A+B, \mathcal{D}(A))$ is closed then $B R(\lambda, A)$ is uniformly ergodic.

Proof. Since the generator of a contraction semigroup is dissipative and densely defined, so is $(A+B, \mathcal{D}(A))$. Thus we have

$$
\operatorname{Im}(\lambda I-\overline{A+B})=\overline{\operatorname{Im}(\lambda I-(A+B))} \text { for all } \lambda>0 .
$$

Hence, $X=\overline{\operatorname{Im}(I-B R(\lambda, A))}$ and the claim follows from Corollary 2.2 and Theorem 2.4, respectively.

Remark 3.4. The assumption in Theorem 3.3 that $B R(\lambda, A)$ is power bounded can be weakened in accordance with Corollary 2.2 and Theorem 2.4.

4. Aspects of positivity. In this section we assume that $X$ is an ordered Banach space with normal and generating cone $X_{+}$. A linear operator $A$ is called resolvent positive if there exists $\omega \in \mathbb{R}$ such that $(\omega, \infty) \subseteq \rho(A)$ and $R(\lambda, A) \geq 0$ for all $\lambda>\omega$. In that case, if $(B, \mathcal{D}(A))$ is a positive operator, then $B R(\lambda, A)$ is an everywhere defined positive linear operator for all $\lambda>\omega$, thus it is bounded. By combining [2, Proposition 3.11.2], [1, Theorem 3.1], [13, Theorem 1.1], and [11, Theorem 4.2] we obtain the following characterization. 
Proposition 4.1. Let $A$ be a resolvent positive operator. Then $s(A)$ $<\infty, s(A) \in \sigma(A)$ when $s(A)>-\infty$, and

$$
R(\lambda, A) \geq R(\mu, A) \geq 0 \quad \text { whenever } \mu>\lambda>s(A) .
$$

Moreover, if $(B, \mathcal{D}(A))$ is a positive operator, then $A+B$ is resolvent positive if and only if $r(B R(\lambda, A))<1$ for some $\lambda>s(A)$, in which case

$$
s(A+B)=\inf \{\lambda>s(A): r(B R(\lambda, A))<1\} .
$$

Corollary 4.2. Let $(A, \mathcal{D}(A))$ be resolvent positive and $(B, \mathcal{D}(A))$ be positive. If $(A+B, \mathcal{D}(A))$ is the generator of a positive $C_{0}$-semigroup, then $B R(\lambda, A)$ is power bounded and quasi-compact for all $\lambda>s(A+B)$.

If $A$ is a generator of a $C_{0}$-semigroup $\{S(t)\}_{t \geq 0}$ then $A$ is resolvent positive if and only if the semigroup $\{S(t)\}_{t \geq 0}$ is positive. However, there are resolvent positive operators which are not generators of $C_{0}$-semigroups [1, Section 3]. We have the following generation result in real Banach lattices.

Lemma 4.3. Let $(A, \mathcal{D}(A))$ be a densely defined resolvent positive operator in a real Banach lattice $X$ and let $\omega \in \mathbb{R}$. Then $(A-\omega I, \mathcal{D}(A))$ generates a $C_{0}$-semigroup of positive contractions if and only if for every $x \in \mathcal{D}(A)_{+}$ there is $x^{*} \in X_{+}^{*}$ with $\left\|x^{*}\right\| \leq 1$ such that $\left\langle x^{*}, x\right\rangle=\|x\|$ and

$$
\left\langle x^{*}, A x\right\rangle \leq \omega\|x\| .
$$

Proof. Suppose first that $(A-\omega I, \mathcal{D}(A))$ is the generator of a $C_{0}$-semigroup $\{S(t)\}_{t \geq 0}$ of positive contractions. Let $x \in \mathcal{D}(A)_{+}$and $x \neq 0$. By the Hahn-Banach theorem, there is $x^{*} \in X_{+}^{*}$ such that $\left\|x^{*}\right\|=1$ and $\left\langle x^{*}, x\right\rangle=$ $\|x\|$ (see e.g. [4, Lemma 3.1]). Since $\left\langle x^{*}, v\right\rangle \leq\|v\|$ for all $v \in X$, we obtain

$$
\begin{aligned}
\left\langle x^{*}, A x\right\rangle-\omega\|x\| & =\left\langle x^{*},(A-\omega I) x\right\rangle=\lim _{t \rightarrow 0} \frac{\left\langle x^{*}, S(t) x\right\rangle-\left\langle x^{*}, x\right\rangle}{t} \\
& \leq \limsup _{t \rightarrow 0} \frac{\|S(t) x\|-\|x\|}{t} \leq 0 .
\end{aligned}
$$

To prove the converse, we make use of the Hille-Yosida theorem. Since $A-\omega I$ is also resolvent positive by the definition, we may assume that $\omega=0$. Since $A$ is resolvent positive, $A$ is closed and the resolvent $R(\lambda, A)$ is a positive operator for all $\lambda>s(A)$, by Proposition 4.1. Thus, it remains to check that $s(A) \leq 0$ and that $\lambda R(\lambda, A)$ is a contraction for all $\lambda>0$. For $x \in \mathcal{D}(A)_{+}$take $x^{*} \in X_{+}^{*}$ with $\left\|x^{*}\right\| \leq 1$ such that $\left\langle x^{*}, x\right\rangle=\|x\|$ and $\left\langle x^{*}, A x\right\rangle \leq 0$. Then, for any $\lambda>0$, we have

$$
\|(\lambda-A) x\| \geq\left\langle x^{*},(\lambda-A) x\right\rangle=\lambda\left\langle x^{*}, x\right\rangle-\left\langle x^{*}, A x\right\rangle \geq \lambda\|x\| .
$$

Consequently, if $\lambda>\max \{0, s(A)\}$ then

$$
\|v\| \geq \lambda\|R(\lambda, A) v\| \quad \text { for all } v \in X_{+},
$$


and $\lambda\|R(\lambda, A)\| \leq 1$, since $R(\lambda, A)$ is positive. Suppose now that $s(A)>0$. Let $\lambda_{n}>s(A)$ and $\lambda_{n} \downarrow s(A)$. We have

$$
\left\|R\left(\lambda_{n}, A\right)\right\| \leq \frac{1}{\lambda_{n}} \leq \frac{1}{s(A)}, \quad n \geq 0 .
$$

Since $s(A)>-\infty$, we obtain $s(A) \in \sigma(A)$, thus $\left\|R\left(\lambda_{n}, A\right)\right\| \rightarrow \infty$, which completes the proof.

Proof of Theorem 1.3. By (G1), $A$ is resolvent positive. Let $\alpha \in(0,1)$ and $C_{\alpha}=A+\alpha B$. Since $B R(\lambda, A)$ is Cesàro bounded, we have $r(B R(\lambda, A))$ $\leq 1$, by Remark 2.3. Thus, $r(\alpha B R(\lambda, A)) \leq \alpha<1$ and the operator $\left(C_{\alpha}, \mathcal{D}(A)\right)$ is resolvent positive, by Proposition 4.1. If $x \in \mathcal{D}(A)_{+}$and $x^{*} \in X_{+}^{*}$, then $\left\langle x^{*}, B x\right\rangle \geq 0$ and we have

$$
\left\langle x^{*}, C_{\alpha} x\right\rangle=\left\langle x^{*},(A+B) x\right\rangle+(\alpha-1)\left\langle x^{*}, B x\right\rangle \leq\left\langle x^{*},(A+B) x\right\rangle .
$$

From (G3) and Lemma 4.3 (applied to $C_{\alpha}$ with $\omega=0$ ) it follows that $\left(C_{\alpha}, \mathcal{D}(A)\right)$ is the generator of a $C_{0}$-semigroup of positive contractions on $X$. Hence $\left\|R\left(\lambda, C_{\alpha}\right)\right\| \leq 1 / \lambda$ for $\lambda>0$, so $\left(C_{\alpha}, \mathcal{D}(A)\right)$ is dissipative and we obtain

$$
\lambda\|x\| \leq\left\|\left(\lambda I-C_{\alpha}\right) x\right\| \leq\|(\lambda I-A-B) x\|+(1-\alpha)\|B x\|
$$

for all $\lambda>0, x \in \mathcal{D}\left(C_{\alpha}\right)=\mathcal{D}(A)$, and $\alpha \in(0,1)$, which shows that the operator $(A+B, \mathcal{D}(A))$ is dissipative. By Theorem 1.1, the closure of this operator generates a $C_{0}$-semigroup of contractions. Similar arguments to those in [4] show, by the Trotter-Kato approximation theorem (see e.g. [5, Theorem 4.8]), that the semigroup is positive.

We conclude this section with the following result which is a consequence of Theorems 1.3, 1.1, Corollary 4.2 and Proposition 4.1.

Corollary 4.4. Let $X$ be a real Banach lattice and let the operators $(A, \mathcal{D}(A))$ and $(B, \mathcal{D}(B))$ satisfy $(\mathrm{G} 1)-(\mathrm{G} 3)$. Then the following are equivalent:

(i) $(A+B, \mathcal{D}(A))$ generates a $C_{0}$-semigroup of positive contractions;

(ii) $r(B R(\lambda, A))<1$ for some $\lambda>0$;

(iii) $B R(\lambda, A)$ is uniformly ergodic for some $\lambda>0$;

(iv) $B R(\lambda, A)$ is power bounded and quasi-compact for some $\lambda>0$.

Acknowledgments. The author would like to thank Michael Lin for interesting discussions. Helpful comments of the anonymous referee are gratefully acknowledged. This work was supported by the Natural Sciences and Engineering Research Council (NSERC, Canada), the Mathematics of Information Technology and Complex Systems (MITACS, Canada), and by Polish MNiSW grant N N201 021133 . This research was partially carried out when the author was visiting McGill University. 


\section{References}

[1] W. Arendt, Resolvent positive operators, Proc. London Math. Soc. 54 (1987), 321349 .

[2] W. Arendt, C. J. K. Batty, M. Hieber and F. Neubrander, Vector-Valued Laplace Transforms and Cauchy Problems, Monogr. Math. 96, Birkhäuser, Basel, 2001.

[3] J. Banasiak and L. Arlotti, Perturbations of Positive Semigroups with Applications, Springer Monogr. Math., Springer, London, 2006.

[4] J. Banasiak and M. Lachowicz, Around the Kato generation theorem for semigroups, Studia Math. 179 (2007), 217-238.

[5] K.-J. Engel and R. Nagel, One-Parameter Semigroups for Linear Evolution Equations, Grad. Texts in Math. 194, Springer, New York, 2000.

[6] G. Frosali, C. V. M. van der Mee and F. Mugelli, A characterization theorem for the evolution semigroup generated by the sum of two unbounded operators, Math. Methods Appl. Sci. 27 (2004), 669-685.

[7] T. Kato, On the semi-groups generated by Kolmogoroff's differential equations, J. Math. Soc. Japan 6 (1954), 1-15.

[8] U. Krengel, Ergodic Theorems, de Gruyter Stud. Math. 6, de Gruyter, Berlin, 1985.

[9] M. Lin, On the uniform ergodic theorem, Proc. Amer. Math. Soc. 43 (1974), 337-340.

[10] G. Lumer and R. S. Phillips, Dissipative operators in a Banach space, Pacific J. Math. 11 (1961), 679-698.

[11] H. R. Thieme, Remarks on resolvent positive operators and their perturbation, Discrete Contin. Dynam. Systems 4 (1998), 73-90.

[12] M. Tyran-Kamińska, Substochastic semigroups and densities of piecewise deterministic Markov processes, J. Math. Anal. Appl. 357 (2009), 385-402.

[13] J. Voigt, On resolvent positive operators and positive $C_{0}$-semigroups on $A L$-spaces, Semigroup Forum 38 (1989), 263-266.

[14] K. Yosida and S. Kakutani, Operator-theoretical treatment of Markoff's process and mean ergodic theorem, Ann. of Math. (2) 42 (1941), 188-228.

Institute of Mathematics

University of Silesia

Bankowa 14

40-007 Katowice, Poland

E-mail: mtyran@us.edu.pl

Received October 2, 2008

Revised version May 9, 2009 